\title{
Discovery of germline-related genes in Cephalochordate amphioxus: A genome wide survey using genome annotation and transcriptome data
}

\author{
Jia-Xing Yue a , Kun-Lung Li ${ }^{\text {b }}$, Jr-Kai Yu ${ }^{\text {b, c, * }}$ \\ ${ }^{a}$ Ecology and Evolutionary Biology, Department of BioSciences, Rice University, 6100 Main \\ Street, Houston TX 77005, USA \\ ${ }^{b}$ Institute of Cellular and Organismic Biology, Academia Sinica, 128 Academia Road, Section 2, \\ Nankang, Taipei, 11529, Taiwan \\ ${ }^{c}$ Institute of Oceanography, National Taiwan University, Taipei, Taiwan \\ *Corresponding author: E-mail jkyu@gate.sinica.edu.tw
}




\begin{abstract}
The generation of germline cells is a critical process in the reproduction of multicellular organisms. Studies in animal models have identified a common repertoire of genes that play essential roles in primordial germ cell (PGC) formation. However, comparative studies also indicate that the timing and regulation of this core genetic program vary considerably in different animals, raising the intriguing questions regarding the evolution of PGC developmental mechanisms in metazoans. Cephalochordates (commonly called amphioxus or lancelets) represent one of the invertebrate chordate groups and can provide important information about the evolution of developmental mechanisms in the chordate lineage. In this study, we used genome and transcriptome data to identify germline-related genes in two distantly related Cephalochordate species, Branchiostoma floridae and Asymmetron lucayanum. Branchiostoma and Asymmetron diverged more than 120 MYA, and the most conspicuous difference between them is their gonadal morphology. We used important germline developmental genes in several model animals to search the amphioxus genome and transcriptome dataset for conserved homologs. We also annotated the assembled transcriptome data using Gene Ontology (GO) terms to facilitate the discovery of putative genes associated with germ cell development and reproductive functions in amphioxus. We further confirmed the expression of 14 genes in developing oocytes or mature eggs using whole mount in situ hybridization, suggesting their potential functions in amphioxus germ cell development. The results of this global survey provide a useful resource for testing potential functions of candidate germlinerelated genes in cephalochordates and for investigating differences in gonad developmental mechanisms between Branchiostoma and Asymmetron species.
\end{abstract}

Keywords: Amphioxus, primordial germ cell, reproduction, development, evolution, GO annotation. 


\section{INTRODUCTION}

The germline cells of metazoan animals are segregated from somatic cell lineages during development either by the inheritance of specific maternal gene products during early cleavages (preformation) or by induction mechanisms that occur during embryogenesis (epigenesis) (Extavour and Akam, 2003; Johnson et al., 2011). Despite the great contrast between these two types of mechanism, once primordial germ cells (PGCs) are specified they usually show distinct morphological features and express a suite of conserved genes, most commonly including Vasa, Nanos, Piwi/Ago, Tudor, Boule/DAZ, and bruno (Juliano and Wessel, 2010; Voronina et al., 2011). Interestingly, many of these genes, including Vasa, Nanos, and Piwi are also expressed in multipotent somatic stem cells, especially in those cells that have great ability to differentiate, proliferate, self-renew and regenerate. These observations have led to the hypothesis that PGCs and somatic stem cells may have evolved from a common pluripotent progenitor cell population that employs a conserved gene regulatory network for its development and maintenance (Juliano et al., 2010; Solana, 2013). Indeed, certain cell types that can produce both gametes and somatic cells, such as the I-cells in Hydra and the archeocytes in sponges (David, 2012; Funayama, 2010), have been identified in early branching metazoan animals, and these conserved genes are expressed in these pluripotent cells. In planarians, neoblasts represent totipotent stem cells that can lead to the regeneration of the entire body (including the germline); these cells also require the expression of a set of similar genes for their formation and normal function (Rink, 2013). Thus, the evolution of diverse germline developmental mechanisms in metazoans may reflect modifications of gene repertoire that interact with this core genetic network as well as changes in the timing or regulatory control to operate this core genetic network in different animals.

The phylogenetic position of Cephalochordata (commonly called amphioxus or lancelets) makes this animal group a useful reference point for studying the evolution of the genome and developmental mechanisms. Currently there is broad consensus that cephalochordates represent the earliest branching group within chordates, and tunicates are the sister group to vertebrates (Bourlat et al., 2006; Delsuc et al., 2006; Delsuc et al., 2008). Compared to tunicates, however cephalochordates retained more ancestral 
chordate characteristics and experienced less genome reorganization or gene loss after they diverged from other chordate lineages (Holland et al., 2008; Holland et al., 2004; Louis et al., 2012; Paps et al., 2012). Moreover, the cephalochordate genome did not undergo the two-round (2R) whole genome duplication events that occurred during early vertebrate evolution (Putnam et al., 2008). Therefore, cephalochordates provide a suitable model for comparing the evolution of genome and of gene function between invertebrate chordates and vertebrates and even between chordates and other metazoan animals (Bertrand and Escriva, 2011).

To study the evolution of germline developmental mechanisms in chordates, our group previously searched the genome of the model amphioxus Branchiostoma floridae (Holland et al., 2008; Putnam et al., 2008) and identified several conserved germline markers including vasa, nanos, piwi, and tudor-domain-containing genes (Wu et al., 2011; Zhang et al., 2013). We showed that transcripts of these genes are maternally deposited in the matured oocytes; after fertilization, these transcripts are asymmetrically localized in the early developing embryos and are subsequently inherited by the specific blastomere that is destined to differentiate into the germline cells in amphioxus (Wu et al., 2011; Zhang et al., 2013). Our results from studies of various Branchiostoma species further suggested that Branchiostoma amphioxus use maternal gene products to specify their germline cells during early embryogenesis (i.e. a preformation mechanism), in contrast to the previous idea that cephalochordates use an inductive mechanism (epigenesis) to form their germ cells (Extavour and Akam, 2003; Extavour, 2007; Frick and Ruppert, 1997). These new findings raised a question regarding the generality of using maternal gene products to specify germline cells in cephalochordates. Expanding our knowledge of the gene complements and mechanisms that control germline specification to cover more distantly related cephalochordate species would facilitate our understanding of how mechanisms for germline specification have evolved in chordates.

In addition to Branchiostoma species, which have paired gonads, there are two other groups of cephalochordates: Epigonichthys and Asymmetron, which have gonads only on the right side (Holland, 2011; Nishikawa, 2004; Ruppert, 1997). Little is known about the biology of Asymmetron and Epigonichthys amphioxus; only recently, researchers successfully obtained laboratory spawning from an Asymmetron amphioxus 
(Asymmetron lucayanum) and described its early development (Holland and Holland, 2010). On the basis of mitochondrial DNA, Epigonichthys was placed as the sister group of Branchiostoma, and Asymmetron was branched outside the Branchiostoma + Epigonichthys clade (Kon et al., 2007; Nohara et al., 2005). According to the molecular clock based on mitochondrial DNA and on nuclear gene sequence data, divergence between Asymmetron and Branchiostoma is estimated to have occurred 120-160 million years ago (MYA), suggesting wide divergence between these two groups (Nohara et al., 2005; Yue et al., 2014).

In this study, we conducted a comprehensive survey of conserved germlinerelated genes in two amphioxus species, Branchiostoma floridae and Asymmetron lucayanum, that represent the two diverged cephalochordate groups. We also searched for potential germline cell developmental genes within the available genome or transcriptome resources in these two species using annotated GO terms. Finally, we tested the validity of our search approach by performing in situ hybridization on $B$. floridae ovarian tissue and mature eggs to examine the expression patterns of a partial list of these identified genes. The identification of these germline-related genes in cephalochordates will contribute to the understanding of the evolution of mechanisms underlying germline specification in chordates and could provide a basis for investigating differences in gonad developmental processes in Branchiostoma and Asymmetron amphioxus. 


\section{MATERIALS AND METHODS}

\subsection{Sequence sources}

B. floridae proteome sequences were downloaded from http://genome.jgipsf.org/Brafl1/Brafl1.home.html following procedures described in Yue et al. 2014. The two sets of A. lucayanum's likely protein-coding transcripts (asymAD and asym20h) were predicted by TransDecoder (http://transdecoder.sourceforge.net) based on the transcriptome assemblies of two pooled A. lucayanum libraries (asymAD for adults and asym20h for larvae) (NCBI BioProject accession SRP035506). The corresponding protein sequences were used as two sets of A. lucayanum proteomes. Details of these two transcriptome assemblies were described in Yue et al. 2014. The proteomes of four wellstudied model organisms, (Drosophila melanogaster, Caenorhabditis elegans, Danio rerio and Mus musculus), as well as those of six other species (Ciona intestinalis, Petromyzon marinus, Latimeria chalumnae, Xenopus tropicalis, Gallus gallus and Homo sapiens) were downloaded from Ensembl (release 78); the proteomes of Trichoplax adhaerens, Amphimedon queenslandica, Nematostella vectensis, Lottia gigantea, Tribolium castaneum and Strongylocentrotus purpuratus were downloaded from EnsemblMetazoa (release 24). The proteome of Callorhinchus milii was downloaded from http://esharkgenome.imcb.a-star.edu.sg/.

\subsection{Identifying conserved germline-related genes in cephalochordates}

We surveyed the existing literature to create a list of known genes that are crucial to the germline cell development process of four well-studied model organisms, Drosophila melanogaster, Caenorhabditis elegans, Danio rerio and Mus musculus (Table S1). We defined these genes as the candidate genes for germline cell development. We identified the representative protein sequence of each gene based on the annotation information provided by Ensembl (www.ensembl.org), FlyBase (http://flybase.org), WormBase (http://www.wormbase.org), ZFIN (www.zfin.org) and MGI (http://informatics.jax.org/). A Pfam (v27.0) search (http://pfam.xfam.org/) was 
performed for each candidate gene to identify its characteristic protein domain structure. For each characteristic domain, we used the hmmsearch program from the hmmer package (v3.1b1) (Eddy. 2011) to search the B. floridae and A. lucayanum proteomes to call out the B. floridae gene models and A. lucayanum transcripts that contain such specific domains based on the hidden Markov model (HMM) pre-built by Pfam (http://pfam.xfam.org/). This provided an inclusive preliminary list for each candidate gene with its potential homologs in the two cephalochordate species.

We then used Muscle (v3.8) (Edgar, 2004) to align the characteristic domain regions and employed FastTree (v2.1.7) (Price et al., 2009) to build the phylogenetic tree. The orthology relationships were inferred based on the topology of the phylogenetic tree. The full domain structure of each complete gene was further checked and compared with the original candidate genes. We also performed BLASTP searches for our candidate genes against the $B$. floridae and $A$. lucayanum proteomes (e-value cutoff = $1 \mathrm{E}-6$ ) to cross-validate our orthology inferences based on the phylogenetic tree. Two candidate genes, bam and $p g c$, lacked any detectable characteristic domains; we therefore directly used the BLASTP result to determine whether there are orthologous genes in cephalochordates. Finally, for all inferred cephalochordate orthologous counterparts of our candidate genes, we performed a BLASTP search against the NCBI nr database to confirm that the top hits were indeed the orthologs of our original candidate genes as a final confirmation of our orthology inference.

To better characterize the genomic structure of piwill genes in cephalochordates, we retrieved the genome assembly, proteome sequences and gene annotation information of B. becheri from http://mosas.sysu.edu.cn/genome/download_data.php (v18h27.r3 version) (Huang et al., 2014). The piwill genes in B. becheri were identified in the same way as their $B$. floridae counterparts. The gene arrangement of piwill gene cluster in $B$. floridae and B. belcheri were plotted using GenomeTools' AnnotationSketch module (Steinbiss et al., 2009). The annotation of neighboring genes around the piwill gene cluster in $B$. floridae and B. becheri were performed by BLASTP searches against Genbank nr database. The acronym names for these neighboring genes were assigned based on the information from the HUGO Gene Nomenclature Committee (HGNC) (http://www.genenames.org). 


\subsection{Identifying potential germline-related genes in cephalochordates by GO annotation}

In Yue et al. 2014, we conducted BLAST2GO annotations for both the B.floridae and A. lucayanum proteomes. In that analysis, we performed a BLASTP search against the SwissProt and NCBI nr databases and used the standard BLAST2GO pipeline (v2.8) (Conesa et al., 2005). In the current study, we screened the B. floridae and A. lucayanum proteomes based on our previous BLAST2GO proteome annotations and called out those B. floridae gene models and A. lucayanum transcripts that are associated with the GO term "germ cell development" (GO:0007281). These cephalochordate gene models/transcripts constituted a second set of germline cell development-related genes in cephalochordates. We also performed Fisher's exact test (Fisher, 1922) with false discover rate (FDR) correction (Benjamini and Hochberg, 1995) to identify GO terms that are overrepresented by these genes against the genomic background.

2.4 Identifying non-redundant B. floridae - A. lucayanum orthologous gene pairs for our identified germline cell development-related genes in cephalochordates.

We employed Proteinortho (v5.11) (Lechner et al., 2011) to identify the orthologous gene/transcript groups within the $B$. floridae proteome and the two $A$. lucayanum proteomes (asymAD and asym20h). Proteinortho made its inference based on reciprocal best hits in all against all BLASTP searches. Within each identified cephalochordate orthologous gene group, if there were members from both the asymAD and asym20h libraries, we selected the longer sequence as the non-redundant representative sequence for A. lucayanum. To further illuminate the orthology relationship, we incorporated all the other species sampled in this study to ensure our orthology inference based on the topology of their joint phylogenetic tree.

\subsection{Retrieving available ESTs/cDNA clones information for our identified B. floridae germ cell development genes}


For our identified $B$. floridae germline cell development-related gene models, we located the available ESTs/cDNA information in both the $B$. floridae cDNA database and the NCBI EST database (Wang et al., 2012; Yu et al., 2008). Using the protein sequence of the corresponding $B$. floridae gene models, we performed a TBLASTN search using an e-value cutoff of 1E-6. When searching against the NCBI EST database, we limited our search organism to $B$. floridae (NCBI taxid: 7739) only.

\subsection{In situ hybridization and image acquisition}

To verify the expression of identified $B$. floridae germline-related genes, cDNA clones identified from the $B$. floridae cDNA database were picked to obtain their plasmid DNA and subsequently used as templates to synthesize digoxigenin (DIG)-labeled antisense RNA probes for in situ hybridization. Template preparation, probe synthesis, and in situ hybridization were performed as previously described (Lu et al., 2012; Wu et al., 2011). Images were obtained using a Zeiss Axio Imager A2 microscope with a Zeiss AxioCam MRc CCD camera. Adobe Photoshop CS4 was used to minimally adjust the brightness of images and Adobe Illustrator CS4 was used to construct the data figure. 


\section{RESULTS AND DISCUSSION}

3.1 Identification of conserved germline-related genes in B. floridae and A. lucayanum by the candidate gene approach

To identify conserved germline-related genes in amphioxus, we compiled a list of candidate genes that have known functions in germline formation and maintenance in four well-studied model organisms (Drosophila melanogaster, Caenorhabditis elegans, Danio rerio and Mus musculus) for our survey (Supplementary Table S1). For each candidate gene compiled in Table S1, we searched for orthologs in the two amphioxus species, B. floridae and A. lucayanum. The major domain that each candidate gene encodes was characterized to facilitate our search. We successfully identified orthologs of nanos, vasa/glh, arrest/bruno/celf2, bgcn/ythdc2, boule/dazl, csul/pmrt5, gcl gld/qki, mael, mago nashi, mex3, orb/cpeb, ovo, prdm1/blimp1, prdm14, pumilio/pufffbf, smB, smD3, smE/snr6 and tsunagi/rmb8a in both amphioxus species (Table 1). The orthologous B. floridae gene models that we identified for nanos and vasa/glh is consistent with previous findings (Wu et al., 2011). Despite an exhaustive search approach, we were unable to find amphioxus orthologs for bam, buc, cxcl12a/sdfla, cxcr4b, dnd1, dppa3/stella, meg, mex, nanog, oma, oskar, pgc, pie, pos1, pou5f1/oct4, and $s m G / s n r 7$, suggesting that amphioxus most likely do not possess orthologs of these genes. Some of these genes, such as the oskar in insects (Ahuja and Extavour, 2014; Ewen-Campen et al., 2012), and the buc, nanog and pou5fl/oct4 in vertebrates (Bontems et al., 2009; Takatori et al., 2008), are likely lineage specific genes and are thus not present in the amphioxus genome. Another possibility is that some of these genes might have diverged too widely to be recognized by standard BLAST and Pfam searches and that more sophisticated search strategies (e.g., (Roch et al., 2014) will be required to retrieve their homologs. Alternatively, amphioxus may have simply lost those genes during evolution. Further analysis will be needed to confirm whether these genes are absent in the amphioxus lineage.

The piwi, dmrt and tdrd genes in our candidate gene list come from Piwiencoding, DM-encoding and TUDOR-encoding multigene families, respectively 

both amphioxus species (Table 2-4). For example, in B. floridae, we found 7 Piwidomain encoding, 8 DM-domain encoding and 16 TUDOR-domain encoding gene models. To further determine the phylogenetic relationship among different homologous gene members, we incorporated 16 other species and constructed phylogenetic trees for each gene family. Figures S1-S3 show the full 18-way phylogenetic trees, and Figures S4-S6 display the downsampled trees. Using this method, some gene members can be confidently assigned to certain orthologous clades annotated by known genes within the clade. For example, B. floridae gene models 126411, 126413 and 91824 should be piwilike 1 whereas the gene models 276092 should be piwi-like 2; the gene model 89999 did not fall into either clade (piwi-like 1 or piwi-like 2) so we defined it as just piwi-like (Table 2 and Figures S1, S4). As reported previously (Zhang et al., 2013), we found that the 3 piwi-like 1 gene models $(126411,126413,91824)$ are co-localized in a $\sim 104.3 \mathrm{~kb}$ region on the same scaffold (Bf_V2_18 on v2.0 assembly or scaffold214 on v1.0 assembly; Figure 1A). We examined this region more carefully by extracting genomic regions including both this $104.3 \mathrm{~kb}$ segment and its flanking $20 \mathrm{~kb}$ regions on both sides for re-annotation to rule out mis-annotation in the original reference genome annotation. We carried out the re-annotation using Softberry's FGENESH program (http://www.softberry.com/berry.phtml?topic=fgenesh) (Salamov and Solovyev, 2000) with $B$. floridae specific gene discovery parameters provided by Softberry. FGENESH reported 4 Piwi-domain-encoding gene models, for which the gene models of 126411 and 91824 are the same as the $B$. floridae reference annotation, whereas the gene model 126413 was divided into 2 individual Piwi-encoding gene models, suggesting that this $104.3 \mathrm{~kb}$ genome segment includes at least 3 and possibly 4 Piwi-gene models. It is known that the process of collapsing two allelic haploid genome copies into one representative copy (e.g., generating the haploid v2.0 B. floridae reference assembly from the diploid v1.0 assembly) is prone to introduce artifacts in the cases in which two allelic gene copies are placed sequentially on the merged scaffold, resulting in a scenario that is indistinguishable from a tandem gene cluster. However, because this $104.3 \mathrm{~kb}$ piwi-like gene cluster is already present on the same scaffold in the v1.0 assembly, the result is unlikely to be due to the haplotype-merging process. Therefore, it is our opinion that this 
$104.3 \mathrm{~kb}$ genome segment more likely represents a true piwi-like gene cluster, a finding that indicates the presence of at least 3 Piwi-like 1 gene models in the B. floridae genome. To further confirm the existence of this piwi-like gene cluster, we examined the piwill gene arrangement in the recently available genome assembly from another amphioxus species, B. becheri (Huang et al., 2014). Interestingly, we found not only one set, but two sets of this syntenic piwill gene clusters in B. becheri (Figure 1B). These two B. belcheri piwill gene clusters are located on the same genomic scaffold (scaffold:178) and the two clusters (one contains three piwill gene models and the other contains two) are roughly $300 \mathrm{~kb}$ apart. These two B. belcheri piwill gene clusters both have similar syntenic structure of the neighboring genes compared to that in B. floridae, although one of them is in opposite direction. This suggests that a further segment duplication might have occurred in B. belcheri.

For the DM-domain- and TUDOR-domain-containing protein families, we identified amphioxus orthologs for $d m r t 1 / a 2, d m r t 2, d m r t 3$ in the DM-domain-encoding gene family (Table 3 and Figures S2, S5) and snd1, stk31, smn, $t d r d 5$, tdrd6, tdrd9 and $t d r d k h$ in the TUDOR-domain-encoding gene family (Table 4 and Figures S3, S6). These phylogenetic classifications were further confirmed by our BLAST search against the NCBI nr database. We also identified other putative DM-domain-encoding genes and TUDOR-domain-encoding genes whose orthologous affiliations cannot be confidently assigned (Table 3 and Table 4; Supplementary Figures S2, and S5). The best hits for these genes in the NCBI nr database are listed in Table 3 and Table 4 as a basis for future detailed characterization.

\subsection{Identifying potential molecular players in the development of amphioxus germ cells by $G O$ annotation}

In addition to our candidate gene approach, we also identified a set of amphioxus gene models/transcripts that may be involved in germline cell development by searching gene models/transcripts that are associated with the GO term "germline-cell development" (GO:0007281) in our previous BLAST2GO annotation. In total, we found 63 such $B$. floridae gene models and 69 such A. lucayanum transcripts from 88 cephalochordate 
orthologous groups. Of these 88 cephalochordate groups, 9 were also identified by the candidate gene approach (Table 5 and Supplementary Table S2). These cephalochordate orthologous groups are tabulated in Table 5, Supplementary Tables S2 and S3. In Table 5, the GO term associations have support from both the $B$. floridae gene models and the $A$. lucayanum transcripts, whereas in Table S2 the GO term association is only supported by the $B$. floridae gene models and in Table S3 the GO term association is supported only by the A. lucayanum transcripts.

We performed Fisher's exact test to determine whether certain molecular function GO terms are also enriched in B. floridae gene models and A. lucayanum transcripts associated with the GO term germline-cell development (GO:0007281). We identified 52 molecular function GO terms that are consistently enriched compared with genomic background across our 3 cephalochordate datasets. The top 10 enriched molecular function GO terms are listed in Table 6 as representatives; the entire list can be found in Supplementary Table S8. Many of the GO terms enriched in this search are related to DNA/RNA-binding functions or to RNA helicase activities, suggesting that many of those genes encode proteins that interact with RNAs. This is consistent with the notion that the products of many germ-cell-specific genes encode evolutionarily conserved proteins that are involved in post-transcriptional gene regulation, especially in regulating the localization, stability, and translational level of mRNAs or interacting with noncoding small RNAs to exert their regulatory functions (Ewen-Campen et al., 2010; Hale et al., 2014; Voronina et al., 2011).

\subsection{Verification of new molecular markers for the amphioxus germ cell development}

For the $B$. floridae germline-related genes that we identified by the candidate gene approach and by the GO term association approach, we further searched for respective cDNA clone/EST hits in the B. floridae cDNA database (Supplementary Tables S4 and S5) and NCBI EST database (Supplementary Tables S6 and S7). It is worth noting that for each query the e-values of the best hits from the two databases are not directly comparable due to difference in the database size. This cDNA/EST information will be very helpful in future functional studies of these genes. 
To determine whether these identified genes are expressed in germline cells, we conducted an initial screening in which representative cDNA clones of 47 genes in Supplementary Tables S5 and S6 were selected and used to synthesize riboprobes. Using whole-mount in situ hybridization of $B$. floridae ovaries and mature eggs, we were able to detect clear expression patterns of 14 genes (Figure 2). The signals were usually more apparent in small oocytes, while the signals in intermediate sized oocytes were mostly diffused and difficult to focus under microscope, likely due to the accumulation of the yolk materials during oogenesis. Thus, in Figure 2 we mostly present images focusing on the smaller oocytes to show the positive in situ hybridization signals in the ovaries. Transcripts of vasa and piwill were detected in both developing oocytes and mature eggs (Fig. 2, B-C, B'-C'); more importantly, these transcripts were asymmetrically localized near the vegetal pole of the unfertilized eggs (Fig. 2, B'-C'). This pattern is consistent with our previous demonstration that vasa and piwill transcripts are components of the vegetal germ plasm in amphioxus eggs (Wu et al., 2011; Zhang et al., 2013). Transcripts of piwil2 could be observed in ovarian tissue and in developing oocytes (Fig. 2D); however, piwil2 transcripts exhibited a uniform distribution pattern in the mature egg (Fig. 2D'). Transcripts of pumilio, mago nashi, mex-3, and one tdrd gene (cDNA cluster 08482) could be detected in developing oocytes (Fig. 2, E-H), but the transcripts of these genes were asymmetrically localized toward the animal pole of the mature egg (Fig. 2, E'-H'). Transcripts of two other $t d r d$ genes (cDNA clusters 15630 and 06837) and of maelstrom were also detected in the ovaries (Fig. 2, I-K), and the transcripts of these genes were localized near the cortex of the animal pole in mature eggs (Fig. 2, I'-K'). Weak transcript signals of another $t d r d$ gene (cDNA cluster 04486) could be detected uniformly distributed in developing oocytes (Fig. 2L), but the signals were localized near the vegetal cortex of the mature egg (Fig. 2L'), similar to the distribution pattern of germ plasm. Weak signals of $s m E / s n r-6$ were also detected uniformly in developing oocytes (Fig. 2M); however, in mature eggs, the signals of smE/snr-6 were localized toward the animal pole (Fig. 2M'). Transcript signals of $b g c n / y t h d c 2$ and another $t d r d$ gene (cDNA cluster 16581) were detected in developing oocytes (Fig. 2, N-O), but no clear evidence of subcellular localization of these transcripts could be observed in the mature egg (Fig. 2, N'-O'). 
In sum, in addition to the three previously described genes vasa, piwill and piwil2, our initial screening demonstrated the expression patterns of 11 novel genes in female germ cells. From our previous studies, we know that the labeling of Vasa and Nanos gene products near the vegetal cortex appear to be co-localized with sheets of electron-dense particles with ER and mitochondria (Wu et al., 2011). The maternal transcripts of the newly identified $t d r d$ (04486) gene were observed distributing in the same pattern near the vegetal cortex (Figure 2L'). As to the transcripts localized near the animal cortex, we suspect they are likely associated with the remnant of the germinal vesicle that is originally in the animal pole of the developing oocyte (Holland and Holland, 1992; Holland and Holland, 1991). Because only ovaries and mature eggs were used in this study, further analysis using male gonadal tissue and embryonic samples will be needed to determine whether the other 33 genes identified in this survey are expressed in testis or during the developmental stages.

\section{CONCLUSION}

In the current study, a comprehensive survey of germline-related genes was conducted using the genome and transcriptome datasets of two amphioxus species. Our computational search resulted not only in the identification of homologs of evolutionary conserved germline-related genes, but also in the discovery of potential candidate genes for germline cell development and function in cephalochordates. In addition, our in situ hybridization demonstrated specific expression patterns of 11 novel genes in B. floridae ovaries and mature eggs, suggesting that these genes likely have some functions in female germ cells. We anticipate that further studies using male germ cells and embryonic samples will provide additional evidence that will enable us to determine the potential functions of the identified genes. The germline-related genes reported here will facilitate future expression analysis and functional studies of the mechanisms of germline development in cephalochordates and should also provide important insights into the evolution of germline specification mechanisms in chordates.

\section{ACKNOWLEDGEMENTS}


J-XY was partially supported by Wray Todd Graduate Fellowship from Rice University. All the computation work of this study was performed at the National Energy Research Scientific Computing Center, which is supported by the Office of Science of the U.S. Department of Energy under Contract No. DE-AC02-05CH11231. Research funds were provided by the Ministry of Science and Technology of Taiwan (101-2923-B-001-004MY2; 102-2311-B-001-011-MY3) and by the Academia Sinica, Taiwan (Career Development Award AS-98-CDA-L06). 


\section{REFERENCES}

Ahuja, A., Extavour, C. G., 2014. Patterns of molecular evolution of the germ line specification gene oskar suggest that a novel domain may contribute to functional divergence in Drosophila. Dev Genes Evol. 224, 65-77.

Benjamini, Y., Hochberg, Y., 1995. Controlling the False Discovery Rate - a Practical and Powerful Approach to Multiple Testing. Journal of the Royal Statistical Society Series B-Methodological. 57, 289-300.

Bertrand, S., Escriva, H., 2011. Evolutionary crossroads in developmental biology: amphioxus. Development. 138, 4819-4830.

Bontems, F., Stein, A., Marlow, F., Lyautey, J., Gupta, T., Mullins, M. C., Dosch, R., 2009. Bucky ball organizes germ plasm assembly in zebrafish. Curr Biol. 19, 414422.

Bourlat, S. J., et al., 2006. Deuterostome phylogeny reveals monophyletic chordates and the new phylum Xenoturbellida. Nature. 444, 85-88.

Conesa, A., Götz, S., García-Gómez, J. M., Terol, J., Talón, M., Robles, M., 2005. Blast2GO: a universal tool for annotation, visualization and analysis in functional genomics research. Bioinformatics. 21, 3674-3676.

David, C. N., 2012. Interstitial stem cells in Hydra: multipotency and decision-making. Int J Dev Biol. 56, 489-497.

Delsuc, F., Brinkmann, H., Chourrout, D., Philippe, H., 2006. Tunicates and not cephalochordates are the closest living relatives of vertebrates. Nature. 439, 965968.

Delsuc, F., Tsagkogeorga, G., Lartillot, N., Philippe, H., 2008. Additional molecular support for the new chordate phylogeny. Genesis. 46, 592-604.

Edgar, R. C., 2004. MUSCLE: multiple sequence alignment with high accuracy and high throughput. Nucleic Acids Res. 32, 1792-1797.

Ewen-Campen, B., Schwager, E. E., Extavour, C. G., 2010. The molecular machinery of germ line specification. Mol. Reprod. Dev. 77, 3-18.

Ewen-Campen, B., Srouji, J. R., Schwager, E. E., Extavour, C. G., 2012. oskar predates the evolution of germ plasm in insects. Curr Biol. 22, 2278-2283.

Extavour, C. G., Akam, M., 2003. Mechanisms of germ cell specification across the metazoans: epigenesis and preformation. Development. 130, 5869-5884.

Extavour, C. G. M., 2007. Evolution of the bilaterian germ line: lineage origin and modulation of specification mechanisms. Integr. Comp. Biol. 47, 770-785.

Fisher, R. A., 1922. On the interpretation of x(2) from contingency tables, and the calculation of P. Journal of the Royal Statistical Society. 85, 87-94.

Frick, J. E., Ruppert, E. E., 1997. Primordial germ cells and oocytes of Branchiostoma virginiae (Cephalochordata, Acrania) are flagellated epithelial cells: Relationship between epithelial and primary egg polarity. Zygote. 5, 139-151.

Funayama, N., 2010. The stem cell system in demosponges: insights into the origin of somatic stem cells. Dev Growth Differ. 52, 1-14.

Hale, B. J., Yang, C. X., Ross, J. W., 2014. Small RNA regulation of reproductive function. Mol Reprod Dev. 81, 148-159. 
Holland, L. Z., et al., 2008. The amphioxus genome illuminates vertebrate origins and cephalochordate biology. Genome Res. 18, 1100-1111.

Holland, L. Z., Holland, N. D., 1992. Early development in the lancelet (= Amphioxus) Branchiostoma floridae from sperm entry through pronuclear fusion - presence of vegetal pole plasm and lack of conspicuous ooplasmic segregation. Biol Bull. 182, 77-96.

Holland, L. Z., Laudet, V., Schubert, M., 2004. The chordate amphioxus: an emerging model organism for developmental biology. Cell Mol Life Sci. 61, 2290-2308.

Holland, N. D., 2011. Spawning periodicity of the lancelet, Asymmetron lucayanum (Cephalochordata), in Bimini, Bahamas. Italian Journal of Zoology. 78, 478-486.

Holland, N. D., Holland, L. Z., 1991. The fine-structure of the growth stage oocytes of a lancelet (= Amphioxus), Branchiostoma Lanceolatum. Invertebr Reprod Dev. 19, 107-122.

Holland, N. D., Holland, L. Z., 2010. Laboratory spawning and development of the Bahama lancelet, Asymmetron lucayanum (cephalochordata): fertilization through feeding larvae. Biol Bull. 219, 132-141.

Huang, S., et al., 2014. Decelerated genome evolution in modern vertebrates revealed by analysis of multiple lancelet genomes. Nat Commun. 5, 5896.

Johnson, A. D., Richardson, E., Bachvarova, R. F., Crother, B. I., 2011. Evolution of the germ line-soma relationship in vertebrate embryos. Reproduction. 141, 291-300.

Juliano, C., Wessel, G., 2010. Versatile germline genes. Science. 329, 640-641.

Juliano, C. E., Swartz, S. Z., Wessel, G. M., 2010. A conserved germline multipotency program. Development. 137, 4113-4126.

Kon, T., Nohara, M., Yamanoue, Y., Fujiwara, Y., Nishida, M., Nishikawa, T., 2007. Phylogenetic position of a whale-fall lancelet (Cephalochordata) inferred from whole mitochondrial genome sequences. BMC Evol Biol. 7, 127.

Lechner, M., Findeiß, S., Steiner, L., Marz, M., Stadler, P. F., Prohaska, S. J., 2011. Proteinortho: detection of (co-)orthologs in large-scale analysis. BMC Bioinformatics. 12, 124.

Louis, A., Roest Crollius, H., Robinson-Rechavi, M., 2012. How much does the amphioxus genome represent the ancestor of chordates? Brief Funct Genomics. $11,89-95$.

Lu, T. M., Luo, Y. J., Yu, J. K., 2012. BMP and Delta/Notch signaling control the development of amphioxus epidermal sensory neurons: insights into the evolution of the peripheral sensory system. Development. 139, 2020-2030.

Nishikawa, T., 2004. A new deep-water lancelet (Cephalochordata) from off Cape Nomamisaki, SW Japan, with a proposal of the revised system recovering the genus Asymmetron. Zoolog Sci. 21, 1131-1136.

Nohara, M., Nishida, M., Miya, M., Nishikawa, T., 2005. Evolution of the mitochondrial genome in cephalochordata as inferred from complete nucleotide sequences from two epigonichthys species. J Mol Evol. 60, 526-537.

Paps, J., Holland, P. W., Shimeld, S. M., 2012. A genome-wide view of transcription factor gene diversity in chordate evolution: less gene loss in amphioxus? Brief Funct Genomics. 11, 177-186. 
Price, M. N., Dehal, P. S., Arkin, A. P., 2009. FastTree: computing large minimum evolution trees with profiles instead of a distance matrix. Mol Biol Evol. 26, 1641-1650.

Putnam, N. H., et al., 2008. The amphioxus genome and the evolution of the chordate karyotype. Nature. 453, 1064-1071.

Rink, J. C., 2013. Stem cell systems and regeneration in planaria. Dev Genes Evol. 223, 67-84.

Roch, G. J., Tello, J. A., Sherwood, N. M., 2014. At the transition from invertebrates to vertebrates, a novel GnRH-like peptide emerges in amphioxus. Mol Biol Evol. 31, 765-778.

Ruppert, E. E., Cephalochordata (Acrania). In: F. W. Harrison, E. E. Ruppert, Eds.), Microscopic Anatomy of Invertebrates, Vol. 15. Wiley-Liss, New York, 1997, pp. 349-504.

Salamov, A. A., Solovyev, V. V., 2000. Ab initio gene finding in Drosophila genomic DNA. Genome Res. 10, 516-522.

Solana, J., 2013. Closing the circle of germline and stem cells: the Primordial Stem Cell hypothesis. EvoDevo. 4, 2.

Steinbiss, S., Gremme, G., Scharfer, C., Mader, M., Kurtz, S., 2009. AnnotationSketch: a genome annotation drawing library. Bioinformatics. 25, 533-534.

Takatori, N., Butts, T., Candiani, S., Pestarino, M., Ferrier, D. E., Saiga, H., Holland, P. W., 2008. Comprehensive survey and classification of homeobox genes in the genome of amphioxus, Branchiostoma floridae. Dev Genes Evol. 218, 579-590.

Voronina, E., Seydoux, G., Sassone-Corsi, P., Nagamori, I., 2011. RNA granules in germ cells. Cold Spring Harb Perspect Biol. 3.

Wang, Y. B., Chen, S. H., Lin, C. Y., Yu, J. K., 2012. EST and transcriptome analysis of cephalochordate amphioxus--past, present and future. Brief Funct Genomics. 11, 96-106.

Wu, H. R., Chen, Y. T., Su, Y. H., Luo, Y. J., Holland, L. Z., Yu, J. K., 2011. Asymmetric localization of germline markers Vasa and Nanos during early development in the amphioxus Branchiostoma floridae. Dev Biol. 353, 147-159.

Yu, J. K., Wang, M. C., Shin, I. T., Kohara, Y., Holland, L. Z., Satoh, N., Satou, Y., 2008. A cDNA resource for the cephalochordate amphioxus Branchiostoma floridae. Dev Genes Evol. 218, 723-727.

Yue, J. X., Yu, J. K., Putnam, N. H., Holland, L. Z., 2014. The transcriptome of an amphioxus, Asymmetron lucayanum, from the Bahamas: a window into chordate evolution. Genome Biol Evol. 6, 2681-2696.

Zhang, Q. J., Luo, Y. J., Wu, H. R., Chen, Y. T., Yu, J. K., 2013. Expression of germline markers in three species of amphioxus supports a preformation mechanism of germ cell development in cephalochordates. EvoDevo. 4, 17. 


\section{FIGURES}

A B. floridae piwil1 cluster on scaffold:Bf_V2_18

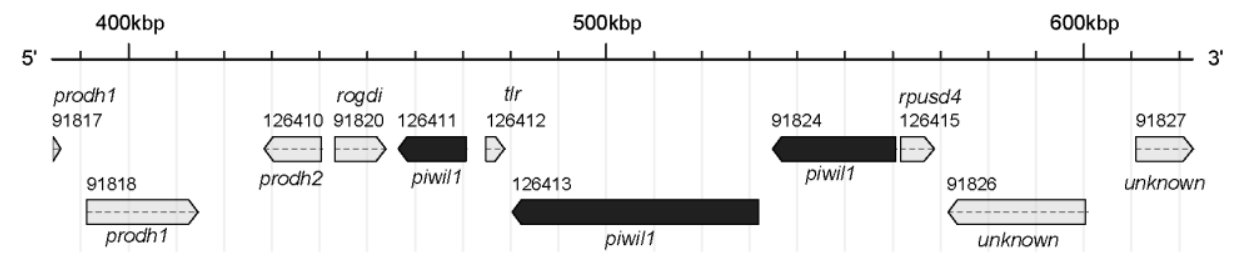

B

B. belcheri piwil1 cluster on scaffold:178

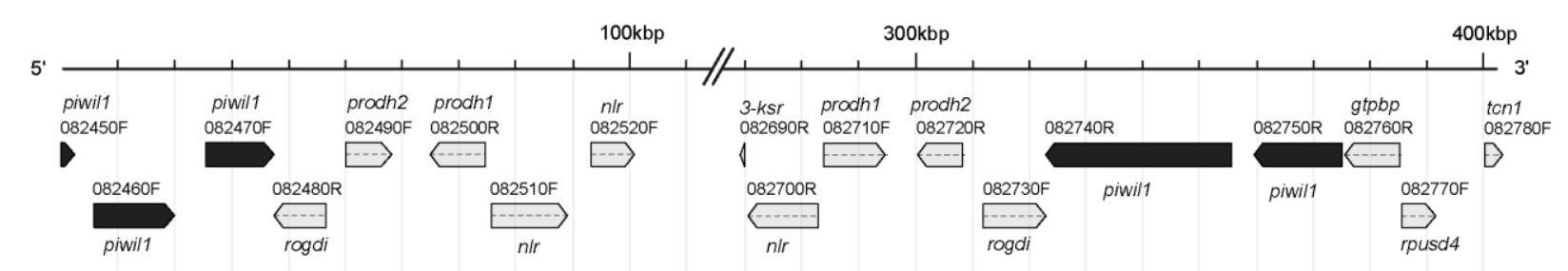

Figure 1. Genomic neighborhoods of piwill gene cluster in B. floridae (A), and the two duplicated piwill gene clusters in B. belcheri (B). The piwill genes are indicated in black color. Annotation of the neighboring genes was inferred based on BLASTP searches against Genbank nr database. 3-ksr: 3-keto-steroid reductase; gtpbp: GTP-binding protein; $n l r$ : NOD-like receptor; prodh1: proline dehydrogenase 1; prodh2: proline dehydrogenase 2; rogdi: rogdi homolog; rpusd4: RNA pseudouridylate synthase domaincontaining protein 4; tcnl: transcobalamin-1; tlr: toll-like receptor. 

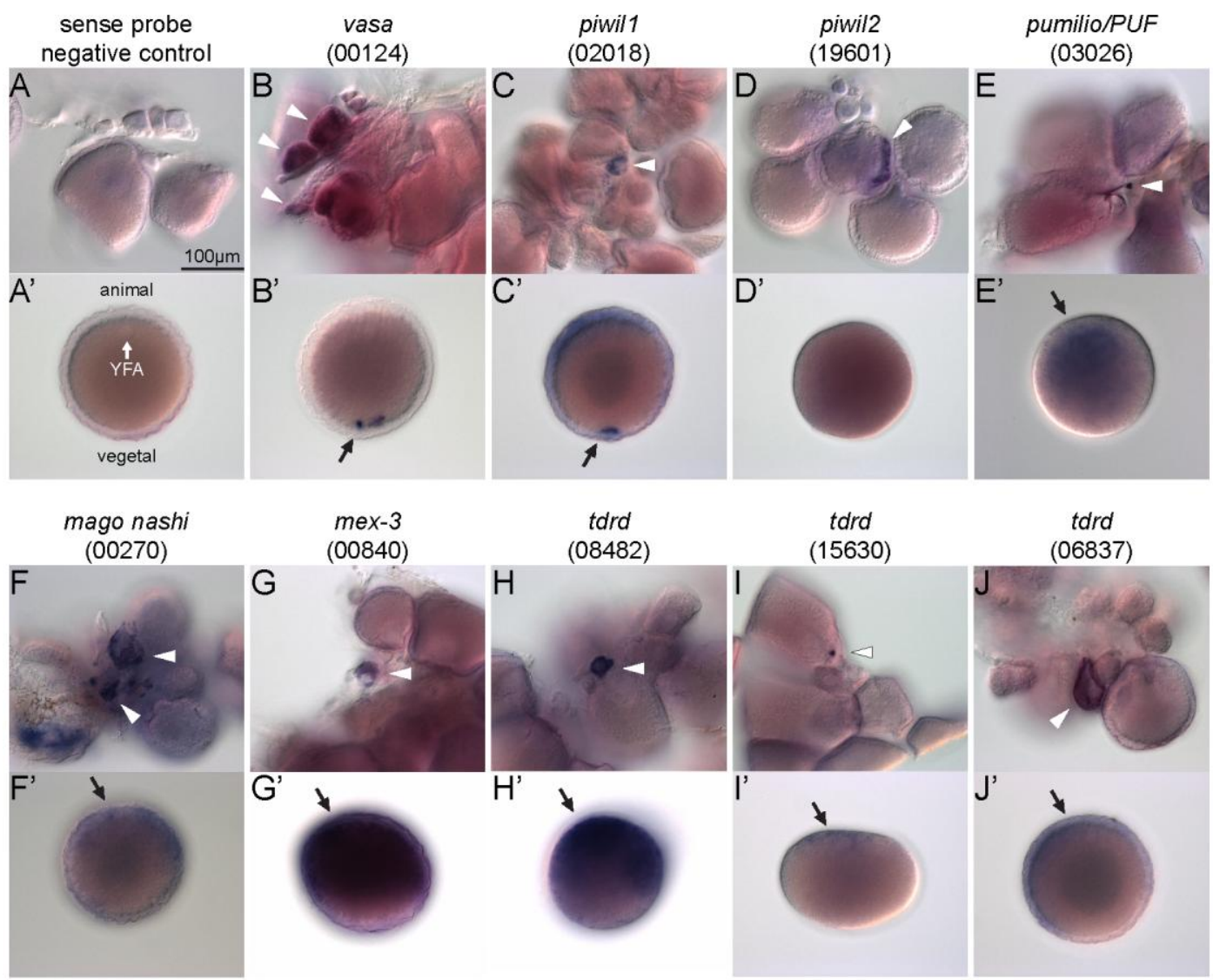

$\operatorname{mex}-3$ (00840)
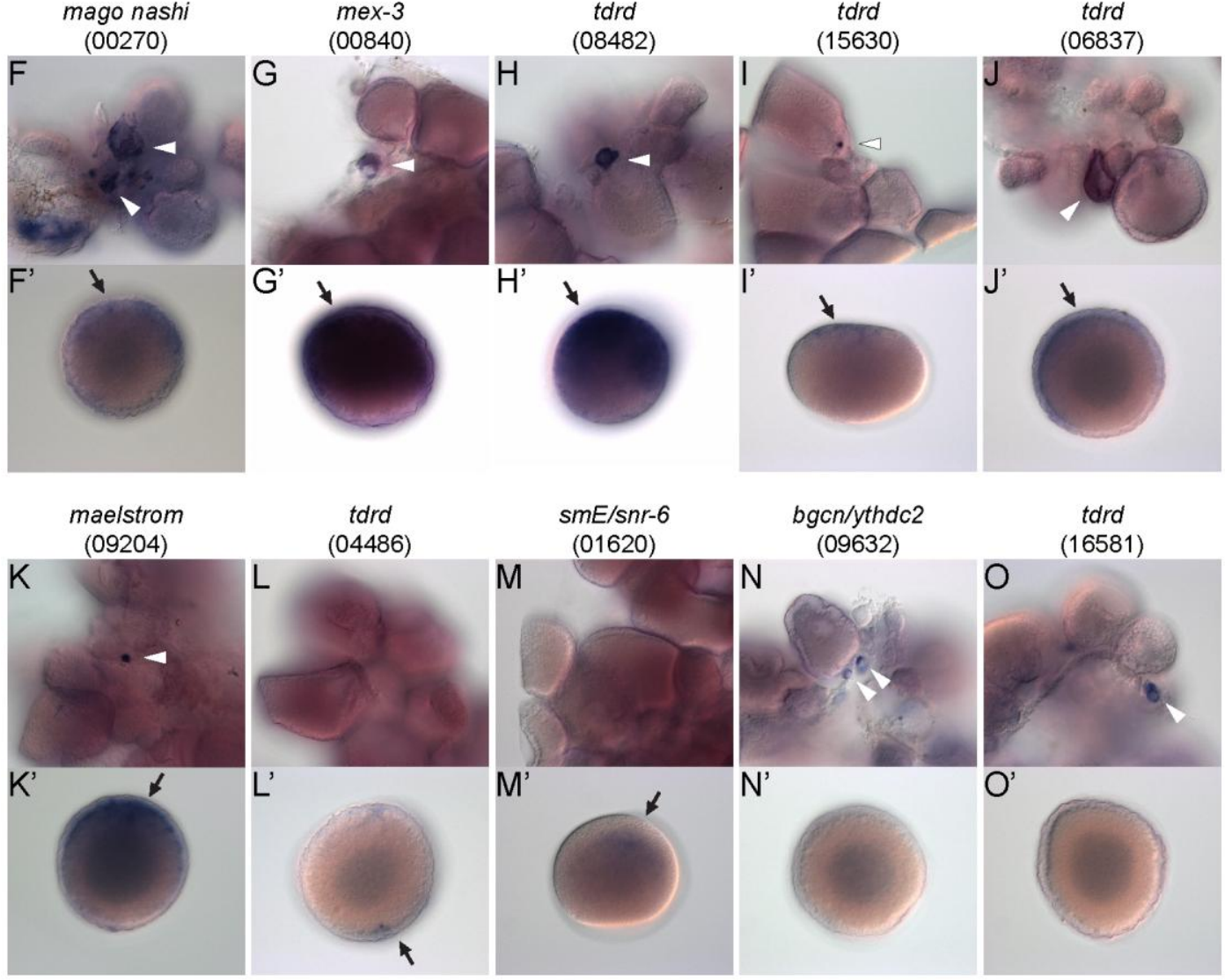

Figure 2. The expression patterns of 14 germline-related genes in B. floridae ovaries and mature eggs detected by whole mount in situ hybridization. A-O, Developing oocytes dissected from female gonads; A'-O', Mature eggs (unfertilized) with the animal pole oriented to the top and vegetal pole to the bottom. (A, A') Sense-riboprobe synthesized from vasa was used as a negative control. (B-O, B'-O') Transcripts of 14 identified genes can be observed in ovaries (white arrows) and/or in mature eggs (black arrows). The white arrow in A' indicates the position of a yolk-free area (YFA), which contains the second meiotic spindle and can be used to recognize the animal pole of the egg. The cDNA cluster ID for anti-sense riboprobe synthesis is indicated below the name of each. The scale bar in $\mathrm{A}$ is $100 \mu \mathrm{m}$, and can be applied to all images. 


\section{TABLES}

Table 1. Amphioxus homologs of conserved genes involved in germline cell development.

\begin{tabular}{llll}
\hline Gene name & Major domain encoded & B.f.gene models & A. l. transcripts (non-redundant) \\
\hline nanos & zf-nanos & $85485^{*}$ & asymAD_comp37714_c0_seq1_m.14292 \\
vasa/glh & DEAD, Helicase_C & $214468^{*}$ & asymAD_comp42457_c0_seq2_m.23103 \\
arrest/bruno/celf 2 & RRM_1 & 114402 & asymAD_comp46076_c0_seq8_m.32483 \\
bgcn/ythdc2 & HA2, OB_NTP_bind & 123593 & asym20h_comp78808_c0_seq2_m.38179 \\
boule/dazl & RRM_1 & 89711 & asym20h_comp73083_c0_seq1_m.25638 \\
csul/pmrt5 & PRMT5 & 89712 & asym20h_comp77866_c0_seq2_m.35437 \\
gcl & BTB & 114443 & asymAD_comp46212_c0_seq1_m.32865 \\
gld/qki & KH_1 & 209210 & asymAD_comp45519_c0_seq3_m.30820 \\
mael & Maelstrom & 236368 & asym20h_comp78683_c0_seq2_m.37777 \\
mago nashi & Mago_nashi & 121310 & asymAD_comp43714_c0_seq1_m.26082 \\
mex3 & KH_1 & 114879 & asymAD_comp46168_c2_seq1_m.32739 \\
orb/cpeb & RRM_1 & 126022 & asymAD_comp15843_c0_seq1_m.716 \\
ovo & zf-C2H2 & 85610 & asymAD_comp38638_c0_seq6_m.15671 \\
prdm1/blimp1 & zf-C2H2 & 226363 & asymAD_comp42374_c0_seq1_m.22917 \\
prdm14 & zf-C2H2 & 120365 & asym20h_comp78929_c0_seq2_m.38578 \\
pumilio/puf/fbf & PUF & 240852 & asym20h_comp79040_c0_seq3_m.38916 \\
smB & LSM & 286861 & asym20h_comp79141_c0_seq2_m.39316 \\
smD3 & LSM & 94837 & asymAD_comp42845_c0_seq2_m.23966 \\
smE/snr6 & LSM & 114009 & asymAD_comp38720_c0_seq3_m.15856 \\
tsunagi/rmb8a & RRM_1 & 268096 & asym20h_comp72130_c1_seq26_m.23962 \\
\hline Footnos: & & 251578 & asym20h_comp66831_c0_seq3_m.15573 \\
\hline
\end{tabular}

Footnotes:

1. Gene models labeled with asterisk (*) were also identified by Wu et al. (2011)

2. We did not find orthologous genes in cephalochordates for bam, buc, cxcl12a/sdfla, cxcr4b, dndl, dppa3/stella, meg, mex, nanog, oma, oskar, pgc, pie, pos1, pou5fl/oct4, smG/snr7 
Table 2. Piwi-encoding gene models/transcripts in B. floridae and A. lucayanum

\begin{tabular}{|c|c|c|c|c|c|c|c|c|c|}
\hline $\begin{array}{l}\text { Gene } \\
\text { name }\end{array}$ & $\begin{array}{l}\text { Major } \\
\text { omain } \\
\text { ncoded }\end{array}$ & $\begin{array}{l}B . f . \text { gene } \\
\text { models }\end{array}$ & A. $l$. transcripts (non-redundant) & Top hit description & Top hit ID & Identity & $\begin{array}{l}\text { E- } \\
\text { value }\end{array}$ & $\begin{array}{l}\text { Bit } \\
\text { score }\end{array}$ & Note \\
\hline \multirow[t]{6}{*}{ piwi } & \multirow[t]{6}{*}{ Piwi } & $126411 *$ & & $\begin{array}{l}\text { PREDICTED: piwi-like protein } \\
1 \text { [Latimeria chalumnae] }\end{array}$ & $\begin{array}{l}\text { ref|XP_00598 } \\
7243.1 \mid\end{array}$ & $54 \%$ & 0 & 943 & \multirow{4}{*}{$\begin{array}{l}\text { piwi- } \\
\text { like }\end{array}$} \\
\hline & & & asymAD_comp47783_c0_seq1_m.3 & Piwi-like 1 protein & $\mathrm{gb} \mid \mathrm{AGI} 95996$ & & & & \\
\hline & & $126413 *$ & 8647 & [Branchiostoma floridae] & $.1 \mid$ & $80 \%$ & 0 & 2262 & \\
\hline & & $91824 *$ & $\begin{array}{l}\text { asym20h_comp66860_c0_seq1_m.1 } \\
5617\end{array}$ & $\begin{array}{l}\text { Piwi-like } 1 \text { protein } \\
\text { [Branchiostoma floridae] }\end{array}$ & $\begin{array}{l}\mathrm{gb} \mid \mathrm{AGI} 95996 \\
.1 \mid\end{array}$ & $87 \%$ & $\begin{array}{r}6.00 \mathrm{E} \\
-74\end{array}$ & 248 & \\
\hline & & $276092 *$ & $\begin{array}{l}\text { asymAD_comp46117_c0_seq1_m.3 } \\
2603\end{array}$ & $\begin{array}{l}\text { Piwi-like } 2 \text { protein } \\
\text { [Branchiostoma floridae] }\end{array}$ & $\begin{array}{l}\mathrm{gb} \mid \mathrm{AGI} 95997 \\
.1 \mid\end{array}$ & $94 \%$ & 0 & 1395 & $\begin{array}{l}\text { piwi- } \\
\text { like } 2\end{array}$ \\
\hline & & $89999 *$ & & $\begin{array}{l}\text { Piwi-like } 2 \text { protein } \\
\text { [Branchiostoma floridae] }\end{array}$ & $\begin{array}{l}\mathrm{gb} \mid \mathrm{AGI} 95997 \\
.1 \mid\end{array}$ & $32 \%$ & $\begin{array}{r}5.00 \mathrm{E} \\
-126\end{array}$ & 408 & $\begin{array}{l}\text { piwi- } \\
\text { like }\end{array}$ \\
\hline \multirow[t]{2}{*}{$\begin{array}{l}\text { argonau } \\
\text { te }\end{array}$} & \multirow[t]{2}{*}{ Piwi } & $77832 *$ & $\begin{array}{l}\text { asymAD_comp42174_c0_seq1_m.2 } \\
2473\end{array}$ & $\begin{array}{l}\text { translation initiation factor } 2 \mathrm{C} \text {, } \\
\text { putative [Ixodes scapularis] }\end{array}$ & $\begin{array}{l}\text { ref|XP_00241 } \\
3188.1 \mid\end{array}$ & $76 \%$ & 0 & 1431 & \\
\hline & & 81672 & $\begin{array}{l}\text { asymAD_comp45264_c0_seq1_m.3 } \\
0080\end{array}$ & $\begin{array}{l}\text { translation initiation factor } 2 \mathrm{C}, \\
\text { putative [Ixodes scapularis] }\end{array}$ & $\begin{array}{l}\text { ref|XP_00241 } \\
3188.1 \mid\end{array}$ & $81 \%$ & 0 & 1422 & \\
\hline
\end{tabular}

Footnote:

1. For each cephalochordate orthologous group, the top hit information was based on the corresponding $B$. floridae gene model (when B. floridae gene model is available) or $A$. lucayanum transcripts (when $B$. floridae gene model is not available)

2. Gene models labeled with asterisk $(*)$ were also identified by Zhang et al. (2013) 
Table 3. DM-encoding gene models/transcripts in B. floridae and A. lucayanum.

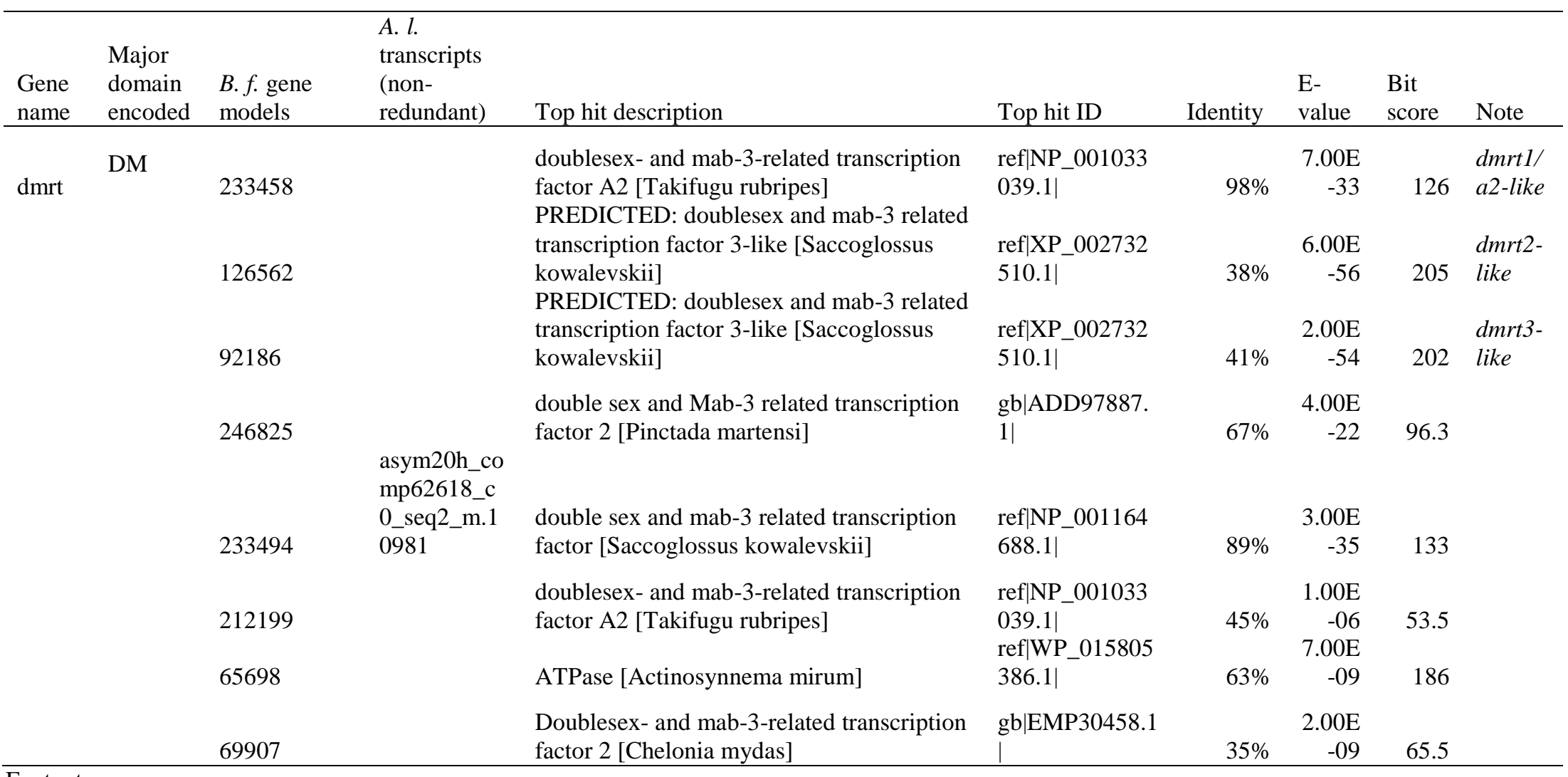

Footnote:

factor 2 [Chelonia mydas]

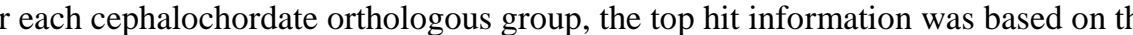

available) or A. lucayanum transcripts (when B. floridae gene model is not available) 
Table 4. TUDOR-encoding gene models/transcripts in B. floridae and A. lucayanum.

\begin{tabular}{|c|c|c|c|c|c|c|c|c|c|}
\hline $\begin{array}{l}\text { Gene } \\
\text { name }\end{array}$ & $\begin{array}{l}\text { Major } \\
\text { domain } \\
\text { encoded }\end{array}$ & $\begin{array}{l}\text { B. } f \text {. gene } \\
\text { models }\end{array}$ & A.l. transcripts (non-redundant) & Top hit description & Top hit ID & $\begin{array}{l}\text { Identi } \\
\text { ty }\end{array}$ & $\begin{array}{l}\text { E- } \\
\text { valu } \\
\mathrm{e}\end{array}$ & $\begin{array}{l}\text { Bit } \\
\text { score }\end{array}$ & Note \\
\hline \multirow[t]{11}{*}{ tdrd } & \multirow[t]{11}{*}{ TUDOR } & $\begin{array}{l}117294 \\
118019\end{array}$ & $\begin{array}{l}\text { asymAD_comp45765_c0_seq1_m. } \\
31529 \\
\text { asymAD_comp47611_c0_seq2_m. } \\
37793\end{array}$ & $\begin{array}{l}\text { PREDICTED: staphylococcal } \\
\text { nuclease domain-containing } \\
\text { protein 1-like [Saccoglossus } \\
\text { kowalevskii] } \\
\text { serine/threonine-protein kinase } \\
31 \text { [Rattus norvegicus] }\end{array}$ & $\begin{array}{l}\text { ref|XP_00273 } \\
2557.2 \mid \\
\text { ref|NP_00117 } \\
8900.1 \mid\end{array}$ & $57 \%$ & $\begin{array}{r}2.00 \\
\text { E-94 } \\
5.00 \\
\text { E-83 }\end{array}$ & 307 & $\begin{array}{l}\text { snd1- } \\
\text { like } \\
\text { stk31- } \\
\text { like }\end{array}$ \\
\hline & & $\begin{array}{l}121736 \\
64001\end{array}$ & $\begin{array}{l}\text { asym20h_comp73447_c0_seq5_m. } \\
26323 \\
\text { asym20h_comp76266_c0_seq1_m. } \\
31846\end{array}$ & $\begin{array}{l}\text { PREDICTED: survival motor } \\
\text { neuron protein [Cavia porcellus] } \\
\text { Tudor domain-containing } \\
\text { protein } 5 \text { [Crassostrea gigas] }\end{array}$ & $\begin{array}{l}\text { ref|XP_00346 } \\
1660.1 \mid \\
\text { gb|EKC3434 } \\
3.1 \mid\end{array}$ & $30 \%$ & $\begin{array}{r}2.00 \\
\mathrm{E}-19 \\
3.00 \\
\mathrm{E}-90 \\
4.00\end{array}$ & $\begin{array}{l}95.5 \\
306\end{array}$ & $\begin{array}{l}\text { smn- } \\
\text { like } \\
\text { tdrd5- } \\
\text { like }\end{array}$ \\
\hline & & 125037 & $\begin{array}{l}\text { asym20h_comp79605_c0_seq1_m. } \\
41219\end{array}$ & $\begin{array}{l}\text { Tudor domain-containing } \\
\text { protein } 1 \text { [Crassostrea gigas] } \\
\text { PREDICTED: putative ATP- } \\
\text { dependent RNA helicase }\end{array}$ & $\begin{array}{l}\mathrm{gb} \mid \mathrm{EKC} 3433 \\
2.1 \mid\end{array}$ & $25 \%$ & $\begin{array}{r}E- \\
116\end{array}$ & 5250 & $\begin{array}{l}\text { tdrd6- } \\
\text { like }\end{array}$ \\
\hline & & 65715 & $\begin{array}{l}\text { asymAD_comp45668_c0_seq1_m. } \\
31259\end{array}$ & $\begin{array}{l}\text { TDRD9-like [Saccoglossus } \\
\text { kowalevskii] } \\
\text { PREDICTED: tudor and KH }\end{array}$ & $\begin{array}{l}\text { ref|XP_00681 } \\
7051.1 \mid\end{array}$ & $49 \%$ & 0 & 1370 & $\begin{array}{l}\text { tdrd9- } \\
\text { like }\end{array}$ \\
\hline & & 67212 & $\begin{array}{l}\text { asym20h_comp74389_c1_seq4_m. } \\
28149 \\
\text { asym20h_comp73791_c0_seq4_m. }\end{array}$ & $\begin{array}{l}\text { domain-containing protein } \\
\text { isoform X5 [Gallus gallus] } \\
\text { tudor domain-containing } 7\end{array}$ & $\begin{array}{l}\text { ref|XP_42340 } \\
0.4 \mid \\
\text { gb|AGI95999 }\end{array}$ & $32 \%$ & $\begin{array}{r}1.00 \\
\mathrm{E}-72\end{array}$ & 266 & $\begin{array}{l}\text { tdrdkh- } \\
\text { like }\end{array}$ \\
\hline & & $125731 *$ & $26914-1-1-1-$ & $\begin{array}{l}\text { protein [Branchiostoma floridae] } \\
\text { Tudor and KH domain- }\end{array}$ & .11 & $96 \%$ & 0 & 2134 & \\
\hline & & 72207 & $\begin{array}{l}\text { asym20h_comp63166_c0_seq6_m. } \\
11486\end{array}$ & $\begin{array}{l}\text { containing protein [Stegodyphus } \\
\text { mimosarum] } \\
\text { PREDICTED: putative ATP- } \\
\text { dependent RNA helicase }\end{array}$ & $\begin{array}{l}\mathrm{gb} \mid \mathrm{KFM} 6285 \\
4.1 \mid\end{array}$ & $33 \%$ & $\begin{array}{r}1.00 \\
\mathrm{E}-32\end{array}$ & 330 & \\
\hline & & & asym20h_comp3999_c0_seq1_m.3 & TDRD12-like [Saccoglossus & ref|XP_00681 & & 8.00 & & \\
\hline & & & 482 & $\begin{array}{l}\text { kowalevskii] } \\
\text { PREDICTED: tudor domain- }\end{array}$ & 4119.1| & $45 \%$ & E-18 & 89.4 & \\
\hline & & 88929 & & $\begin{array}{l}\text { containing protein 1-like } \\
\text { [Saccoglossus kowalevskii] }\end{array}$ & $\begin{array}{l}\text { ref|XP_00682 } \\
4064.1 \mid \\
\text { gb|EKC4288 }\end{array}$ & $31 \%$ & $\begin{array}{r}7.00 \\
\text { E-93 }\end{array}$ & 549 & \\
\hline & & 120982 & 7228 & protein 12 [Crassostrea gigas] & 7.1 & $31 \%$ & E- & 717 & \\
\hline
\end{tabular}


PREDICTED: tudor domainasymAD_comp47682_c0_seq1_m.

116968 38082

containing protein 7

[Orycteropus afer afer]

PREDICTED: tudor domain-

asymAD_comp46963_c0_seq1_m. containing protein 1 [Latimeria 35225

chalumnae

PREDICTED: putative leucine-

rich repeat-containing protein

asym20h_comp68196_c0_seq2_m.

DDB_G0290503-like

[Saccoglossus kowalevskii]

A kinase anchor protein 1,

asymAD_comp47341_c0_seq7_m. mitochondrial [Crassostrea 36652

gigas]

Schlafen-like protein 1

[Zootermopsis nevadensis]

PREDICTED: tudor domain-

asym20h_comp73843_c0_seq1_m. containing protein 7-like

27012

[Saccoglossus kowalevskii]

PREDICTED: RING finger

asym20h_comp68194_c0_seq2 m. protein 17 isoform X7 [Mus 17619 musculus]

Tudor domain-containing

asymAD_comp44373_c1_seq1_m. protein 6 [Tauraco

27575

erythrolophus]

asym20h_comp23968_c0_seq1_m. Tudor domain-containing

987 protein 1 [Pelecanus crispus]

asym20h_comp55522_c0_seq1_m. Tudor domain-containing

7225 protein 12 [Crassostrea gigas]

asymAD_comp47812_c0_seq1_m. RING finger protein 17

38821

[Crassostrea gigas]

PREDICTED: putative leucine-

asymAD_comp45215_c0_seq2_m. rich repeat-containing protein

29922

[Saccoglossus kowalevskii]

asym20h_comp30294_c0_seq1_m.

1746

tudor domain-containing protein, putative [Ixodes scapularis]

asym20h_comp59727_c0_seq1_m.

PING finger protein 17 [Zootermopsis nevadensis]

\begin{tabular}{|c|c|c|c|}
\hline ref|XP_00794 & & 1.00 & \\
\hline $5180.1 \mid$ & $29 \%$ & E-57 & 273 \\
\hline ref|XP_00600 & & 3.00 & \\
\hline 2877.1| & $28 \%$ & E-61 & 506 \\
\hline ref|XP_00681 & & 1.00 & \\
\hline 1719.1| & $27 \%$ & E-12 & 83.6 \\
\hline $\mathrm{gb} \mid \mathrm{EKC} 1806$ & & 4.00 & \\
\hline $6.1 \mid$ & $53 \%$ & E-55 & 196 \\
\hline gb|KDR0968 & & 8.00 & \\
\hline $3.1 \mid$ & $33 \%$ & E-09 & 67.8 \\
\hline ref|XP_00682 & & 3.00 & \\
\hline $1058.1 \mid$ & $27 \%$ & E-50 & 194 \\
\hline ref|XP_00651 & & 3.00 & \\
\hline 9175.1| & $30 \%$ & E-46 & 296 \\
\hline $\mathrm{gb} \mid \mathrm{KFV} 0967$ & & 2.00 & \\
\hline $6.1 \mid$ & $25 \%$ & E-81 & 1635 \\
\hline $\mathrm{gb} \mid \mathrm{KFQ} 5907$ & & 1.00 & \\
\hline $3.1 \mid$ & $48 \%$ & E-09 & 100 \\
\hline $\mathrm{gb} \mid \mathrm{EKC} 4288$ & & 6.00 & \\
\hline 7.1 & $37 \%$ & E-48 & 223 \\
\hline $\mathrm{gb} \mid \mathrm{EKC} 1907$ & & & \\
\hline $1.1 \mid$ & $34 \%$ & 0 & 778 \\
\hline ref|XP_00681 & & 3.00 & \\
\hline 1719.1| & $39 \%$ & E-10 & 76.3 \\
\hline ref|XP_00240 & & 7.00 & \\
\hline $8421.1 \mid$ & $31 \%$ & E-08 & 57.8 \\
\hline $\mathrm{gb} \mid \mathrm{KDR} 1474$ & & 8.00 & \\
\hline 9.1| & $31 \%$ & E-17 & 241 \\
\hline
\end{tabular}


Footnote:

1. For each cephalochordate orthologous group, the top hit information was based on the corresponding $B$. floridae gene model (when $B$. floridae gene model is available) or $A$. lucayanum transcripts (when $B . f$. gene model is not available).

2. Gene models labeled with asterisk (*) were also identified by Zhang et al. (2013).

Table 5. Cephalochordate-orthologous groups in which both B. floridae gene models and A. lucayanum transcripts are associated with the GO term germline-cell development (GO:0007281).

\begin{tabular}{|c|c|c|c|c|c|c|c|}
\hline \multirow{2}{*}{$\begin{array}{l}B . f . \text { gene } \\
\text { models }\end{array}$} & \multirow[b]{2}{*}{ A. $l$. transcripts (non-redundant) } & \multicolumn{6}{|c|}{ Top hits in NCBI_nr database by the corresponding $B . f$. gene model } \\
\hline & & Top hit description & Top hit ID & Identity & $\begin{array}{l}\text { E- } \\
\text { value }\end{array}$ & $\begin{array}{l}\text { Bit } \\
\text { score }\end{array}$ & Note \\
\hline 85485 & $\begin{array}{l}\text { asymAD_comp37714_c0_seq1_m.14 } \\
292 \\
\text { asym20h_comp77866_c0_seq2_m.3 }\end{array}$ & $\begin{array}{l}\text { nanos-like protein [Branchiostoma } \\
\text { floridae] } \\
\text { protein boule-like [Otolemur }\end{array}$ & $\begin{array}{l}\text { gb|ADM26639.1| } \\
\text { ref|XP_003803032. }\end{array}$ & $100 \%$ & 0 & 587 & $\begin{array}{l}\text { nanos } \\
\text { boulel }\end{array}$ \\
\hline 89712 & 5437 & garnettii] & $1 \mid$ & $45 \%$ & $3 \mathrm{E}-28$ & 122 & $\begin{array}{l}d a z l \\
p r d m\end{array}$ \\
\hline 120365 & $\begin{array}{l}\text { asym20h_comp78929_c0_seq2_m.3 } \\
8578 \\
\text { asymAD_comp42457_c0_seq2_m.23 }\end{array}$ & $\begin{array}{l}\text { blimp1 [Branchiostoma floridae] } \\
\text { vasa-like protein [Branchiostoma }\end{array}$ & $\mathrm{gb}|\mathrm{ACH} 72078.1|$ & $96 \%$ & 0 & 1434 & $\begin{array}{l}1 / b l i \\
m p 1\end{array}$ \\
\hline 214468 & 103 & floridae] & $\mathrm{gb}|\mathrm{AGI} 96004.1|$ & $100 \%$ & 0 & 764 & vasa \\
\hline 268096 & $\begin{array}{l}\text { asym20h_comp72130_c1_seq26_m. } \\
23962\end{array}$ & $\begin{array}{l}\text { Small nuclear ribonucleoprotein E } \\
\text { [Dicentrarchus labrax] } \\
\text { PREDICTED: PR domain zinc }\end{array}$ & emb|CBN80607.1| & $88 \%$ & $\begin{array}{l}8.00 \mathrm{E}- \\
52\end{array}$ & 168 & $\begin{array}{l}s m E / s \\
n r-6\end{array}$ \\
\hline 240852 & $\begin{array}{l}\text { asym20h_comp79040_c0_seq3_m.3 } \\
8916 \\
\text { asym20h_comp73083_c0_seq1_m.2 }\end{array}$ & $\begin{array}{l}\text { finger protein 14-like [Saccoglossus } \\
\text { kowalevskii] } \\
\text { PREDICTED: protein boule-like }\end{array}$ & $\begin{array}{l}\text { ref|XP_002739286. } \\
2 \mid \\
\text { ref|XP_006818817. }\end{array}$ & $86 \%$ & $\begin{array}{l}0 \\
1.00 \mathrm{E}-\end{array}$ & 617 & $\begin{array}{l}\text { prdm } \\
14 \\
\text { boulel }\end{array}$ \\
\hline 89711 & 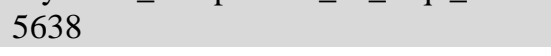 & [Saccoglossus kowalevskii] & $1 \mid$ & $37 \%$ & 33 & 140 & dazl \\
\hline 115963 & $\begin{array}{l}\text { asymAD_comp42235_c0_seq1_m.22 } \\
637\end{array}$ & $\begin{array}{l}\text { PREDICTED: U2 small nuclear } \\
\text { ribonucleoprotein A'-like } \\
\text { [Lepisosteus oculatus] } \\
\text { PREDICTED: serine/threonine- }\end{array}$ & $\begin{array}{l}\text { ref|XP_006628826. } \\
1 \mid\end{array}$ & $73 \%$ & $\begin{array}{l}7.00 \mathrm{E}- \\
123\end{array}$ & 362 & \\
\hline 230456 & $\begin{array}{l}\text { asym20h_comp59976_c0_seq1_m.9 } \\
111\end{array}$ & $\begin{array}{l}\text { protein kinase Chk2-like [Aplysia } \\
\text { californica] }\end{array}$ & $\begin{array}{l}\text { ref|XP_005112692. } \\
1 \mid\end{array}$ & $65 \%$ & $\begin{array}{l}5.00 \mathrm{E}- \\
166\end{array}$ & 485 & \\
\hline
\end{tabular}


PREDICTED: probable ATP-

2006 asymAD_comp40204_c0_seq1_m.18 dependent RNA helicase DHX40 503 asymAD_comp37090_c0_seq1_m.13 protein 5 isoform X2 [Poecilia

$125404 \quad 397$ asymAD_comp39341_c0_seq1_m.16

265042

898

12238

asymAD_comp44568_c2_seq1_m.28

089

1003797880

asymAD_comp45660_c1_seq $8 \_m .31$ 240

80164

280395

asymAD_comp46055_c1_seq1_m.32 423

59675

asymAD_comp46357_c0_seq1_m.33 314

asym20h_comp72765_c0_seq5_m.2

$117669 \quad 5104$

RNA helicase DHX15-like isoform

asymAD_comp45366_c0_seq1_m.30 Full=Microtubule-associated protein 361

68495

asymAD_comp36658_c0_seq1_m.12

264344 856

asym20h_comp76525_c0_seq1_m.3

$71433 \quad 2413$

asymAD_comp43709_c3_seq1_m.26

$113775 \quad 078$

asymAD_comp47154_c1_seq2_m.35

205455 isoform X2 [Pelodiscus sinensis]

PREDICTED: bone morphogenetic reticulata

small nuclear ribonucleoprotein D2-

like protein [Azumapecten farreri]

Ras

microsporus

PREDICTED: bcl-2 homologous

antagonist/killer-like

[Strongylocentrotus purpuratus]

PREDICTED: DNA mismatch

repair protein Msh2 [Saccoglossus

kowalevskii]

PREDICTED: putative pre-mRNA-

splicing factor ATP-dependent

X2 [Aplysia californica]

PREDICTED: pre-mRNA-splicing

factor ATP-dependent RNA

helicase PRP16-like [Xiphophorus maculatus]

RecName: Full=Targeting protein for Xklp2 homolog; Short=TPX2 homolog; AltName:

TPX2 homolog [Patiria pectinifera]

ref|XP_006138715.

1) $61 \% \quad 0$

ref|XP_008428025. 1.00E-

1) $55 \% \quad 101$

$1.00 \mathrm{E}-$

gb|AAM94277.1| $96 \% \quad 74$

ref|NP_001133260.

1) $78 \% \quad 119$

$6.00 \mathrm{E}-$

emb|CEG81615.1| $41 \% \quad 29$

7.00E-

ref|XP_793197.1| $51 \% \quad 46$

ref|XP_002740239.

$1 \mid$

$71 \%-0$

ref|XP_005092201.

1) $86 \% \quad 0$

1263

ref|XP_005810482.

1)

$71 \% \quad 0$

1785

sp|E2RYF8.1|TPX

2_PATPE

ref|NP_001004780.

$1 \mid$

$46 \%$

$5.00 \mathrm{E}-$

147

$3.00 \mathrm{E}-$

D1 [Xenopus (Silurana) tropicalis]

PREDICTED: H/ACA

ref|XP_007889523.

gb|AAK84395.1|A

F397147_1

$77 \% \quad 0$

685

4 [Callorhinchus milii]

belcheri]

ref|XP_006825596.

$1 \mid$

PREDICTED: superkiller viralicidi activity 2-like 2-like [Saccoglossus
$100 \%$

$6.00 \mathrm{E}-$

100

295

$80 \% \quad 0$ 


$\begin{array}{ll} & \text { asymAD_comp45593_c0_seq2_m.31 } \\ 66988 & 047 \\ & \text { asymAD_comp38746_c1_seq3_m.15 } \\ & 889 \\ 143690 & \text { asymAD_comp47769_c0_seq2_m.38 } \\ 124682 & 566 \\ 221588 & \text { asymAD_comp43940_c0_seq2_m.26 } \\ & 599\end{array}$

86149

asymAD_comp47778_c0_seq1_m.38 624

281075

asymAD_comp39577_c0_seq1_m.17 313 asymAD_comp45149_c1_seq7_m.29 737

116859

63599 asymAD_comp45723_c0_seq1_m.31 417

202253

asym20h_comp70989_c0_seq1_m.2 1996 asymAD_comp35738_c0_seq1_m.11

130314 614

124728

asymAD_comp42509_c0_seq1_m.23 212

asymAD_comp41171_c0_seq1_m.20

260605 351

asymAD_comp46344_c0_seq2_m.33

$277402 \quad 274$ asymAD_comp46023_c0_seq5_m.32 247509 337 asymAD_comp40636_c1_seq1_m.19 99541 ubiquitin-conjugating enzyme E2 [Penaeus monodon] PREDICTED: glutamate receptorinteracting protein 1 isoform $\mathrm{X} 1$ [Callorhinchus milii]

PREDICTED: U5 small nuclear

ribonucleoprotein $200 \mathrm{kDa}$ helicase [Orycteropus afer afer]

PREDICTED: activin receptor type1 [Caprimulgus carolinensis] PREDICTED: serine/threonine-

protein kinase mTOR

[Callorhinchus milii]

PREDICTED: U6 snRNA-

associated Sm-like protein LSm4

[Diaphorina citri]

retinoic acid receptor beta,isoform 4

[Gallus gallus]

nucleostemin-like protein

[Saccoglossus kowalevskii]

PREDICTED: mediator of RNA

polymerase II transcription subunit 6 [Callorhinchus milii]

PREDICTED: inhibitor of growth protein 1 [Chrysochloris asiatica] PREDICTED: putative ATPdependent RNA helicase DHX33 [Strongylocentrotus purpuratus] PREDICTED: gamma-secretase subunit PEN-2-like

[Strongylocentrotus purpuratus] PREDICTED: SNW domain-

containing protein 1-like

[Saccoglossus kowalevskii]

Bcl-2-like protein 1 [Crassostrea gigas]

PREDICTED: helicase SKI2W [Anolis carolinensis]

\begin{tabular}{|c|c|c|c|}
\hline $\mathrm{gb}|\mathrm{ACD} 13594.1|$ & $88 \%$ & $\begin{array}{l}5.00 \mathrm{E}- \\
103\end{array}$ & 307 \\
\hline $\begin{array}{l}\text { ref|XP_007903149. } \\
1 \mid\end{array}$ & $53 \%$ & 0 & 1089 \\
\hline $\begin{array}{l}\text { ref|XP_007957626. } \\
1 \mid \\
\text { ref|XP } 010162237\end{array}$ & $80 \%$ & 0 & 3665 \\
\hline $1 \mid$ & $68 \%$ & 0 & 624 \\
\hline $\begin{array}{l}\text { ref|XP_007895970. } \\
1 \mid\end{array}$ & $75 \%$ & 0 & 2144 \\
\hline $\begin{array}{l}\text { ref|XP_008476479. } \\
\text { 1| }\end{array}$ & $75 \%$ & $\begin{array}{l}7.00 \mathrm{E}- \\
56\end{array}$ & 181 \\
\hline $\begin{array}{l}\text { emb|CAA40615.1| } \\
\text { ref|NP 001161618. }\end{array}$ & $61 \%$ & $\begin{array}{l}3.00 \mathrm{E}- \\
161\end{array}$ & 473 \\
\hline $1 \mid$ & $52 \%$ & 0 & 634 \\
\hline $\begin{array}{l}\text { ref|XP_007891615. } \\
1 \mid \\
\text { ref|XP } 006851578 .\end{array}$ & $66 \%$ & $\begin{array}{l}3.00 \mathrm{E}- \\
108 \\
3.00 \mathrm{E}-\end{array}$ & 324 \\
\hline & $52 \%$ & 85 & 268 \\
\hline ref|XP_792543.2| & $63 \%$ & $\begin{array}{l}1.00 \mathrm{E}- \\
139\end{array}$ & 422 \\
\hline ref|XP_791533.1| & $63 \%$ & $\begin{array}{l}2.00 \mathrm{E}- \\
43\end{array}$ & 147 \\
\hline
\end{tabular}

ref|XP_002741369.

2

$\mathrm{gb|EKC30554.1|}$ ref|XP_003218045.

1)
0

$9.00 \mathrm{E}-$

28

$2.00 \mathrm{E}-$
089

665

604 
PREDICTED: putative pre-mRNAsplicing factor ATP-dependent

90438 asymAD_comp46262_c0_seq1_m.33 042

RNA helicase DHX15-like [Oryzias latipes]

ubiquitin-conjugating enzyme e2

$78802 \quad \begin{aligned} & \text { asymAD_comp42095_c1_seq1_m.22 } \\ & 295\end{aligned}$ d4-like [Lichtheimia corymbifera JMRC:FSU:9682]

PREDICTED: CUGBP Elav-like asym20h_comp76564_c0_seq10_m. family member 4 [Callorhinchus

89423 32490

milii]
ref|XP 004079718.

1) $43 \% \quad 0$

mb $|\mathrm{CDH} 5607.1| \quad 32 \%$

17

82.8

ref|XP_007895323. $\quad$ 8.00E-

\section{Footnote:}

1

$68 \%$

70

The highlighted gene models were also identified by our candidate gene approach.

Table 6. Enriched molecular function GO terms for $B$. floridae gene models associated with the GO term germline-cell development (GO:0007281).

\begin{tabular}{clc}
\hline GO ID & \multicolumn{1}{c}{ GO description } & FDR* \\
\hline GO:1901363 & heterocyclic compound binding & $3.69 \mathrm{E}-18$ \\
GO:0097159 & organic cyclic compound binding & $1.11 \mathrm{E}-17$ \\
GO:0003724 & RNA helicase activity & $6.36 \mathrm{E}-14$ \\
GO:0003676 & nucleic acid binding & $9.95 \mathrm{E}-14$ \\
GO:0004386 & helicase activity & $1.97 \mathrm{E}-12$ \\
GO:0000166 & nucleotide binding & $2.89 \mathrm{E}-12$ \\
GO:1901265 & nucleoside phosphate binding & $2.90 \mathrm{E}-12$ \\
GO:0008026 & ATP-dependent helicase activity & $1.23 \mathrm{E}-11$ \\
GO:0070035 & purine NTP-dependent helicase activity & $1.23 \mathrm{E}-11$ \\
GO:0035639 & purine ribonucleoside triphosphate binding & $1.44 \mathrm{E}-11$ \\
\hline
\end{tabular}

Footnote:

$\operatorname{FDR}(*)$ : false discovery rate. 